\title{
Beam Sensitivity in EPMA: The Analysis of Apatite, $\mathrm{Ca}_{5}\left(\mathrm{PO}_{4}\right)_{3}(\mathrm{~F}, \mathrm{Cl}, \mathrm{OH})$
}

\author{
Carl E. Henderson
}

Department of Geological Sciences, University of Michigan, Ann Arbor, MI 48109

Microanalysts have long recognized that many geological materials, including carbonates, feldspars, zeolites, and natural glasses are sensitive to the electron beam, exhibiting compositional change during analysis through sample degradation, element volatilization or migration, or phase transformation. Even in his doctoral thesis, Raymond Castaing was concerned about these phenomena and calculated the thermal effect caused by the concentration of an electron beam into a small sample volume [1]. Methods used to mitigate sample damage and decrease x-ray count rate variation include the use of reduced beam current, lower (or higher) beam voltage, increased beam size (either by electron beam spot rastering or defocusing), beam-blanking intervals, various metal coatings for increased thermal conductivity, sample cooling, and sample motion during analysis [25]. Each of these methods has drawbacks which may limit its applicability to a given sample or analytical requirement. Reduced beam intensities result in lower analytical precision, unless counting times are concurrently increased, while beam broadening or sample motion techniques negate true "micro"-analysis, though large area analysis may be acceptable in some cases.

Time scans, or plots of x-ray count rate versus beam exposure time, are essential tools for evaluating the suitability of analytical conditions on beam-sensitive materials. Even if x-ray temporal variation cannot be completely eliminated by one of the methods mentioned above, it may be possible to find conditions where the count rate can be predictably extrapolated back to an initial, or time-zero, count rate by measuring a series of sub-counting periods at the beginning of analysis [6-7].

Apatite, $\mathrm{Ca}_{5}\left(\mathrm{PO}_{4}\right)_{3}(\mathrm{~F}, \mathrm{Cl}, \mathrm{OH})$, is a common accessory mineral found in a wide variety of geological settings. The proportion of $\mathrm{F}, \mathrm{Cl}$ and $\mathrm{OH}$ in apatite gives important clues about the fluid conditions during mineral formation, but since $\mathrm{H}$ cannot be measured directly by EPMA, accurate measurement of $\mathrm{F}$ and $\mathrm{Cl}$ is essential for calculation of $\mathrm{OH}$. Stormer et al. demonstrated crystallographicallycontrolled anisotropy of $\mathrm{F}$, and to a lesser extent, $\mathrm{Cl}$, x-ray count rates during analysis of apatite [8]. In their study, samples oriented with the crystallographic c-axis parallel to the electron beam showed greater x-ray variation with time than samples oriented with the c-axis perpendicular to the beam, with large $\mathrm{F}$ count rate increases during initial beam exposure.

A suite of oriented apatite samples was examined on two CAMECA EMPAs at the University of Michigan. Time scans were acquired over a range of beam currents and beam sizes in an attempt to find conditions yielding stable, or predictably varying, $\mathrm{F}$ and $\mathrm{Cl}$ count rates. Even at beam currents $\leq 10 \mathrm{nA}$ or beam diameters $\geq 10 \mu \mathrm{m}$, the extrapolated time-zero $\mathrm{F}$ count rate was systematically higher for samples oriented with the c-axis parallel to the beam than for samples with the beam normal to the c-axis (Fig. 1). Various methods used for microanalysis of other beam-sensitive materials were also evaluated on apatite, with cryogenic sample cooling (Fig. 2) and sample motion during analysis showing the greatest promise in eliminating the difference in $\mathrm{F}$ concentrations measured on the two sample orientations. Without the use of these additional techniques, however, analysis of apatite grains of unknown orientation remains problematic, especially for F-rich apatite, and the analyst should be aware of the distinct possibility of overestimating $\mathrm{F}$ concentration. If 
possible, the $\mathrm{F}$ count rate should be monitored during analysis for use in time-zero count rate extrapolation or for outright analysis rejection [9].

References

[1] R. Castaing, University of Paris (1951).

[2] G.B. Morgan, VI and D. London, Amer. Mineral. 90 (2005) 1131.

[3] C.H. Nielsen and H. Sigurdsson, Microbeam Analysis (1980) 139.

[4] M.P. Smith, J. Sed. Petrol. 56 (1986) 560.

[5] S.L. Kearns et al., Microsc. Microanal. 8 (Suppl. 2) (2002) 1562CD.

[6] C.H. Nielsen and H. Sigurdsson, Amer. Mineral. 66 (1981) 547.

[7] J.D. Devine et al., Amer. Mineral. 80 (1995) 319.

[8] J.C. Stormer, Jr. et al., Amer. Mineral. 78 (1993) 641.

[9] F.M. McCubbin et al., 40 $0^{\text {th }}$ Lunar and Planetary Sci. Conf. (2009) 2246.

[10] The author gratefully thanks his collaborators Dr. Youxue Zhang and Karen Wang of the University of Michigan for supplying the oriented apatite samples and dedicates this talk to the memory of Eric J. Essene, who mentored a generation of microprobe users at Michigan.
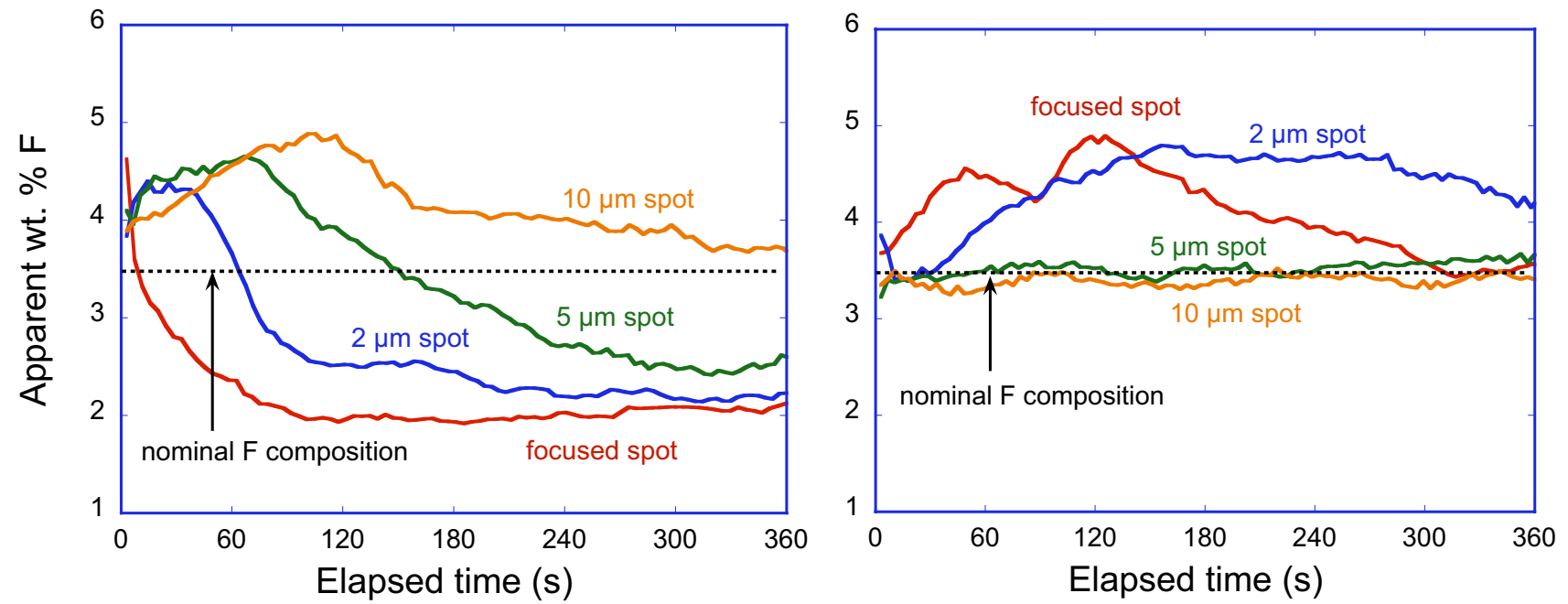

FIG. 1. Time scans from Durango apatite measured with a $15 \mathrm{kV}, 10 \mathrm{nA}$ beam at various beam spot sizes. Graph on left is from a sample oriented with the c-axis parallel to the electron beam; graph on right is from a sample oriented with the c-axis perpendicular to the beam.

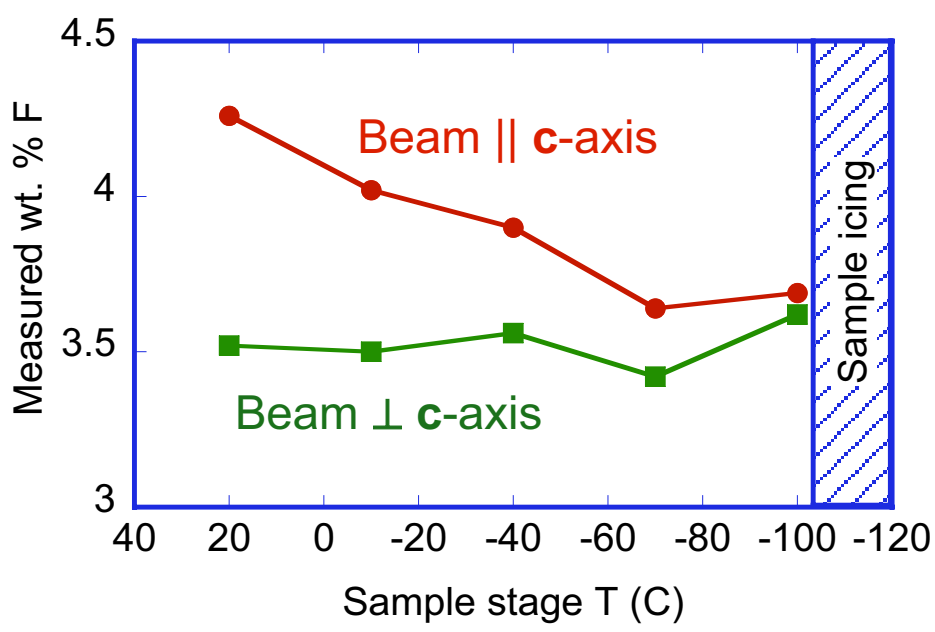

FIG. 2. Measured concentrations on Wilberforce fluorapatite in two orientations as a function of sample temperature. Below $-100 \mathrm{C}$, ice formed on the surface of the sample. 